\title{
Corela
}

Cognition, représentation, langage

HS-8 | 2010

L'interpellation

\section{Interpellation et nomination en milieu religieux orthodoxe}

\section{Felicia Dumas}

\section{OpenEdition}

\section{Journals}

Édition électronique

URL : http://journals.openedition.org/corela/733

DOI : $10.4000 /$ corela.733

ISSN : $1638-573 \mathrm{X}$

\section{Éditeur}

Cercle linguistique du Centre et de I'Ouest - CerLICO

Référence électronique

Felicia Dumas, «Interpellation et nomination en milieu religieux orthodoxe », Corela [En ligne], HS-8 | 2010, mis en ligne le 01 octobre 2010, consulté le 20 avril 2019. URL : http:// journals.openedition.org/corela/733; DOI : 10.4000/corela.733

Ce document a été généré automatiquement le 20 avril 2019

\section{(c) (i) (2)(2)}

Corela - cognition, représentation, langage est mis à disposition selon les termes de la licence Creative Commons Attribution - Pas d'Utilisation Commerciale - Partage dans les Mêmes Conditions 4.0 International. 


\title{
Interpellation et nomination en milieu religieux orthodoxe
}

\author{
Felicia Dumas
}

1 Nous nous proposons de montrer de quelle façon les différentes formes d'interpellation et de nomination employées à l'intention et à l'égard des différents acteurs monastiques, des prélats, des clercs, et des simples fidèles dans l'orthodoxie mettent en scène les rôles religieux à l'intérieur d'une hiérarchie ecclésiastique et monastique, et, respectivement, les rôles liturgiques, lors de la célébration des offices de l'Eglise. Nos références porteront sur les termes employés dans l'orthodoxie française.

\section{Corpus}

2 Ces termes ont été recensés lors d'un travail de compulsation d'un corpus de plus de quarante types de sources religieuses écrites, telles : des textes liturgiques proprement dits (les offices de l'orthodoxie), des ouvrages de catéchèse ou de spiritualité orthodoxes, des livres de théologie, des petits lexiques orthodoxes rédigés en français, des revues, etc. Une autre partie du corpus est représentée par des enregistrements vidéo de quelques offices orthodoxes (la liturgie eucharistique et les vêpres), et audio d'une dizaine d'entretiens réalisés avec des acteurs très initiés de l'orthodoxie (française): quelques évêques ${ }^{1}$, le supérieur d'un monastère de moines ${ }^{2}$, la mère supérieure d'un monastère de femmes $^{3}$, de simples fidèles, des moines et des moniales orthodoxes qui pratiquent leur religion en France et l'expriment en langue française.

Moins connue que d'autres confessions chrétiennes, à cause de son implantation plutôt récente dans l'espace de l'Hexagone, l'orthodoxie française s'est développée une terminologie spécifique, propre à l'individualiser à l'intérieur du paradigme religieux chrétien, et surtout à la distinguer de sa grande "sur" confessionnelle, la religion catholique. Cette terminologie est constituée -certes- d'un noyau dur d'origine latine, auquel s'ajoutent de nombreux emprunts à la langue grecque ${ }^{4}$, considérée comme langue liturgique par excellence de l'orthodoxie en général. Le contact entre le français et le grec s'est produit en France, par l'intermédiaire de l'acte de traduction (des traductions 
massives des textes liturgiques représentatifs de l'ensemble de l'orthodoxie), ainsi que par le contact direct entre les locuteurs d'origine grecque -qui parlaient le grec- et les locuteurs français, convertis à l'orthodoxie. ${ }^{5}$ C'est aussi pour cette raison de nouveauté que nous nous rapporterons aux interpellations employées dans ce milieu religieux orthodoxe (monastique y compris).

\section{Méthodologie}

Nous analyserons ces différentes formes d'interpellation et de nomination employés à l'égard de ces acteurs (religieux et laïques) orthodoxes au niveau de leurs emplois différents dans:1. des fragments de discours et des types d'interaction verbales enregistrées (offices liturgiques, rencontres en dehors des offices), et 2.des textes écrits de factures différentes (où ils sont plutôt nommés : des lettres monastiques, des ouvrages de catéchèse, et d'autres). Nous étudierons également le fonctionnement de l'interpellation dans le déclenchement de certains rôles liturgiques du prêtre, lors de la célébration de la liturgie eucharistique par exemple (la messe de l'Eglise orthodoxe): lorsque le chœur prononce l'exhortation "Père saint bénit", le prêtre accomplit certains actes très précis de paroles et/ou gestuels lors de cet office. De la même façon, on essaiera d'analyser le fonctionnement de quelques formes d'auto-nomination du prêtre en tant qu'acteur liturgique, qui engendrent également une mise en scène de son rôle liturgique.

\section{Nomination et interpellation : la mise en discours énoncé du langage, ou « la conversion du langage en discours ${ }^{6}$}

5 L'une des définitions proposées pour l'interpellation est celle de fonction énonciative et pragmatique des termes d'adresse, qui servent à désigner l'interlocuteur. ${ }^{7}$ Par termes d'adresse, les auteurs comprennent " l'ensemble des expressions dont le locuteur dispose pour désigner son allocutaire (ou destinataire direct) ${ }^{8}{ }^{8}$ Ils sont à distinguer des appellatifs, qui peuvent désigner, en plus de l'allocutaire, aussi bien le délocuté (la personne dont on parle), voire le locuteur. ${ }^{9}$ Parmi ces-derniers, il y aurait des appellatifs "passe-partout ", tels : Monsieur, Madame, Mademoiselle, qu'ils considèrent comme faisant partie des noms d'adresse (sous-catégorie des termes d'adresse), à côté des noms propres (prénoms et / ou nom de famille, diminutifs et surnoms), des termes de parenté, des titres, des termes de profession, des termes affectueux ou injurieux..$^{10}$ D'autres linguistes appellent tout simplement cette sous-catégorie de termes d'adresse, des appellatifs. ${ }^{11} \mathrm{La}$ nomination pourrait être définie -quant à elle- comme la fonction dénominative des appellatifs et des pronoms d'adresse, servant à mettre en évidence, à marquer les relations interpersonnelles, en fixant l'identité de la personne concernée, définie forcément par rapport à l'initiateur de la nomination. La nomination se manifeste au niveau du discours énoncé, tandis que l'interpellation relève de l'énonciation, du langage énoncé en situation d'interlocution. Les deux procédés linguistiques sont des marqueurs de la relation interpersonnelle, engendrée par le contexte socioculturel, par le cadre énonciatif. 


\subsection{Interpellation et nomination à l'œuvre dans la reconnaissance et la définition des rôles religieux à l'intérieur d'une hiérarchie}

6 L'étude de l'interpellation relève donc d'une approche énonciative et pragmatique du discours où les termes d'adresse et les pronoms à fonction interlocutive sont employés à l'égard des différents acteurs religieux de la confession orthodoxe. Commençons par les acteurs monastiques. L'orthodoxie ne connaît qu'un seul ordre monastique, tant pour les hommes que pour les femmes. Dans ce milieu monastique, les moines et les moniales entre eux se servent de quelques interpellations très précises, qui représentent autant de "signes" d'acceptation de jouer leur rôle de membres d'une communauté basée sur des rapports assez codifiés du point de vue hiérarchique: l'interpellation est constituée du nom "sœur", ou, respectivement, "père" (pour les moines), suivi du prénom monastique de ces acteurs, reprise par une autre forme, qui va dans le même sens de la reconnaissance d'un statut égal à l'intérieur d'un système hiérarchisé: ta fraternité, qui est une forme d'interpellation habituelle entre des moines du même "degré" monastique ${ }^{12}$. Quant à la nomination directe ou indirecte, elle est pratiquement identique, sans comprendre pour autant la formule «ta fraternité ». Toujours à l'intérieur du système monastique, au niveau d'une interaction hiérarchique ascendante ${ }^{13}$, le supérieur d'un monastère orthodoxe (l'higoumène) est interpellé par les moines qui vivent dans son monastère (en milieu orthodoxe français d'influence grecque) par l'appellatif géronda,du grec géronүép $\omega v$ (vieux), sans le nom propre, forme identique à la forme de nomination de ce supérieur. De la même façon, la mère supérieure d'un monastère féminin sera interpellée par les moniales qui vivent sous sa direction spirituelle et administrative par

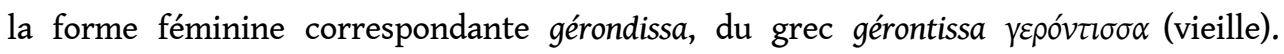
Comme la communauté monastique en question ne se rapporte spirituellement qu'à un seul père (respectivement, une seule mère) supérieure, l'emploi du prénom de celui-ci (celle-ci) est superflue. Au niveau d'une interaction hiérarchique descendante, l'higoumène d'un monastère interpellera ses moines (et, respectivement, ses moniales) par leurs prénoms monastiques ; néanmoins, l'interpellation qu'il utilisera par rapport à des moines plutôt âgés (ou, en tout cas, plus âgés que lui) est formée de l'appellatif père, suivi de son prénom monastique, interpellation reprise dans l'interaction en question par une autre forme, -ta fraternité. Pour s'adresser lors d'une interaction communicationnelle du même type que la précédente (hiérarchique descendante, occasionnée par des rencontres quotidiennes dans l'enceinte du monastère), à des sœurs plus âgées qu'elle, l'higoumène d'un monastère de femmes se servira d'une interpellation formée de l'appellatif sœur, suivi du prénom monastique. L'usage d'employer l'autre interpellation, ta fraternité, entre les moniales, est quasiment inexistant. Le pronom d'adresse employé sera, dans tous les cas, celui de la deuxième personne du singulier : tu. Ce dernier trahit une certaine façon de comprendre et d'exprimer la relation interpersonnelle dans ce milieu religieux très particulier et très radical (du point de vue de leur choix de vivre à l'écart du monde, dans la pauvreté, l'obéissance et la chasteté), de la vie monastique. Nous nous rapportons ici aux communautés monastiques caractérisées par une vie cénobitique, car il n'y a pas en France de monastères orthodoxes à vie idiorrythmique. ${ }^{14}$ Les moines et les moniales orthodoxes qui vivent dans un monastère dirigé par un père (ou une mère) supérieur (e) sont tous des frères dans leur religion et dans leur vie de "famille " spirituelle, une vie qu'ils ont choisie délibérément d'embrasser. A l'époque des Pères de l'Eglise, le mot frère désignait tout chrétien, et le mot fratrie, l'Eglise chrétienne, dans son 
ensemble. Ensuite, à partir du IIIème siècle, le mot frère commence à servir d'appellatif entre les clercs et il engendre un système de parenté spirituel hiérarchisé en pères, fils (ou enfants spirituels) et frères spirituels. De nos jours, la notion de fratrie se rapporte surtout aux communautés monastiques cénobitiques. ${ }^{15}$

7 En ce qui concerne les autres formes d'interpellations employées à l'égard des moines et des moniales orthodoxes, elles sont actualisées par les fidèles (orthodoxes, autrement dit, initiés dans l'emploi correct des appellatifs et des termes d'adresse), donc par des interlocuteurs qui proviennent de l'extérieur du système monastique. Ceux-ci s'adresseront à un moine par l'appellatif père suivi de son prénom monastique, et à une moniale par l'appellatif sœur, suivi de son prénom monastique. Une différence intervient au niveau de la forme de l'interpellation qui vise la mère supérieure, qui sera appelée à intervenir dans une interaction par l'appellatif mère, suivi du prénom monastique de celle-ci. Les plus initiés des fidèles s'adresseront même à celle-ci par une formule qui est très recherchée, et qui reflète la connaissance et la reconnaissance de sa position hiérarchique, révérende mère (ou, très révérende mère Hypandia). Cette dernière interpellation est plutôt caractéristique des interactions cérémoniales, lors des cérémonies liturgiques, ou des conférences (synaxes). Autrement, elle est ressentie plutôt comme prétentieuse. Par contre, en ce qui concerne le père supérieur, celui-ci sera interpellé par les fidèles initiés par la formule révérend père (ou très révérend père, s'il est archimandrite, et dans la plupart des cas, il l'est). Et nous voici arrivée à un autre aspect concernant les relations interpersonnelles et les différentes formes d'interpellation des moines et des moniales orthodoxes, à savoir les différents degrés hiérarchiques qui existent dans ce milieu : nous avons déjà vu le cas de l'archimandrite. Il $\mathrm{y}$ a également des moines de grand schème (les plus avancés spirituellement), de petit schème (ou microschème), des novices et des postulants (par ordre décroissant de la hiérarchie). Même si connues par les fidèles, ces différences hiérarchiques ne sont pas reflétées par des formes d'interpellation spéciales. On les retrouve consignées, en revanche, par les nominations. Quant aux fidèles, ils seront interpellés par des moines et des moniales (y compris par les higoumènes) par leur noms de baptême, cette forme d'interpellation trahissant aussi la relation de paternité définie par rapport à ceux-ci, dont on parlait plus haut. Ces dernières formes d'interpellation (avec des fidèles) sont actualisées lors des interactions plutôt occasionnelles, lors des séjours des fidèles dans des monastères orthodoxes, et leur participation à des offices, à des travaux, à des réunions de catéchèse, etc. Elles intègrent, du point de vue lexico-sémantique, le même champ référentiel des termes de parenté, qui désignent des membres d'une famille spirituelle : père, mère, sœur, frère. Voyons maintenant ce qu'il en est des nominations employées dans ce milieu.

8 Nous en avons déjà mentionné quelques unes concernant la façon dont les moines et les moniales se désignent entre eux (formées des noms sœur et, respectivement, père suivis du prénom monastique). Les nominations que ceux-ci utilisent à l'égard du supérieur de leur monastère sont identiques aux interpellations: géronda, et, respectivement, gérondissa. Ces derniers les nomment par leurs prénoms monastiques, précédés parfois de l'appellatif de parenté spirituelle, sœur et, respectivement, père. Ces nominations sont présentes dans plusieurs types de sources : un discours religieux plutôt métalinguistique, d'initiation et d'explication de ces termes corrects d'adresse (des glossaires ${ }^{16}$ et des livres de catéchèse), ou bien, un discours religieux proprement-dit, qui est caractéristique de la littérature de spiritualité orthodoxe. Pour désigner un vieux père (moine), très avancé 
dans la vie spirituelle, il y a également la nomination indirecte l'Ancien, qu'on rencontre dans des sources très particulières, de la deuxième catégorie, à savoir des textes de la littérature de sagesse spirituelle, présentés sous la forme des biographies des pères spirituels. ${ }^{17}$ Une autre forme de nomination est représentée par le nom abba (en araméen "père »), qui est employé en même temps que sa forme francisée -abbé-, pour désigner un père spirituel, un moine avancé sur la voie de la perfection spirituelle : «L'abba n'est pas un supérieur au sens canonique du mot. C'est avant tout un homme pacifié, libéré de ses passions, et qui par son seul rayonnement communique déjà aux autres quelque chose de l'Esprit qu'il porte en lui ${ }^{18} \mathrm{Si}$ du point de vue sémantique, il n'y a pas de différence entre les deux formes de nominations, du point de vue de l'usage, la forme abbé est suivie toujours du prénom monastique de la personne ainsi désignée, tandis que la forme abba se suffit à elle-même, étant employée sans le nom du moine désigné : «D'abord moine à Scété, l'abbé Isaïe fonda dans la suite un monastère près de Gaza $»{ }^{19}$ Les deux formes sont néanmoins employées pour désigner des vieux moines qui se trouvent aux origines du monachisme oriental, n'étant plus reprises à l'égard des moines orthodoxes de l'époque contemporaine. En revanche, une autre forme de nomination, d'origine slavonne cette fois-ci, est employée pour désigner un moine «qui mène une vie de contemplation d'union intime avec Dieu- et assure une direction spirituelle $"{ }^{20}$ à savoir starets ${ }^{21}$ (avec le pluriel mentionné par les auteurs des sources qui le proposent -startsy). ${ }^{22}$ Elle apparait notamment comme marque linguistique caractéristique des textes de littérature religieuse d'influence culturelle slave, ou russe en particulier.

En ce qui concerne les prélats, les exemples d'interpellations, ainsi que de nominations, sont beaucoup plus complexes. Cette complexité est donnée, en premier lieu, par l'intervention du pronom d'adresse. Les prélats de l'Eglise orthodoxes sont en quelque sorte l'équivalent des prélats majeurs investis de l'épiscopat de l'Eglise romane: le patriarche, les métropolites, les archevêques et les évêques. Commençons par les nominations, pour voir ensuite leur transformation en interpellations, au niveau de leur récupération énonciative par des interlocuteurs, lors des échanges communicatifs, actualisés dans des cadres énonciatifs très précis. L'emploi de ces nominations est une preuve de la reconnaissance et de l'acceptation de leur statut hiérarchique très élevé par la communauté ecclésiastique. Elles sont constituées du déterminant possessif neutre, de la troisième personne -attribuée à celui qui n'est pas présent de façon conversationnelle dans le discours- et d'un nom (désignant une caractéristique qualificative "exceptionnelle") qui le situe hiérarchiquement, du fait d'une codification unanimement reconnue par cette communauté bine définie (des orthodoxes français), à l'intérieur d'un groupe à part de "personnages-acteurs", investis sacramentellement (par une consécration) et symboliquement, à l'intérieur d'une hiérarchie acceptée par l'actualisation même de ces formes de nominations. Pour le patriarche œcuménique, la nomination employée est Sa Sainteté, suivie du prénom de celui-ci; pour le patriarche d'une l'église orthodoxe, on emploie la formule Sa Béatitude, suivi de son prénom; pour les métropolites et les archevêques, la forme de nomination est constituée du titre honorifique son éminence, suivie d'un autre titre "passe-partout" -Monseigneur-, de son prénom, et reprise par le nom de sa dignité ecclésiastique : "Son Eminence Monseigneur Séraphin, métropolite d'Allemagne et d'Europe du Centre et du Nord"; pour les évêques et les évêques vicaires, la structure de la nomination est la même, seuls changent le titre honorifique (son excellence) et le nom de sa dignité ecclésiastique: "Son Excellence Monseigneur Marc, évêque vicaire de la Métropole Orthodoxe Roumaine d'Europe Occidentale et Méridionale". Ces nominations sont consignées dans des types particuliers 
de textes: des lettres pastorales, des récits d'intronisation épiscopales ou métropolitaines ou d'ordination à la prêtrise, des messages de félicitation ou de condoléances, prononcés pour des enterrements: "Extrait du discours de Sa Sainteté Bartholomé Ier pour l'enterrement de Sa Béatitude Théoctiste". ${ }^{23}$ Ou bien: "Dimanche 23 juillet a eu lieu l'ordination à la prêtrise du hiérodiacre Nectarie Petre... A cet événement ont participé Son Eminence Monseigneur Joseph, métropolite de l'Europe Occidentale et méridionale, Son Eminence Mgr. Séraphin, archevêque d'Ottawa et Canada, Son Excellence Mgr. Galaction, évêque d'Alexandria et Son Excellence Mgr. Ciprian, évêque vicaire patriarcal". ${ }^{24}$ Nous avons là exclusivement les formes de nominations employées par des acteurs religieux ou laïques qui ont un statut inférieur, subordonné, par rapport aux statuts hiérarchiques de ces prélats. Dans ce cas précis d'interaction hiérarchique ascendante non actualisée de façon énonciative, on a pu remarquer une marque de l'irruption de la subjectivité des acteurs dénominateurs (auteurs de la nomination), dans la forme de la nomination, subjectivité (de nature affective) exprimée par le déterminant possessif notre : «Quatre années après l'intronisation de Son Eminence Mgr. Nicolas comme archevêque de l'Archevêché Orthodoxe Roumain des Etats- Unis et du Canada par Sa Béatitude notre PèreThéoctiste,les Roumains des Etats- Unis et du Canada se sont de nouveau rassemblés... pour participer à l'ordination comme évêque -vicaire de l'Archimandrite Jean-Cassien $»{ }^{25}$ C'est toujours dans ce cas d'interaction et de types de textes, que l'emploi du pluriel de la forme de nomination est permis (ainsi que, parfois, l'abréviation S.E.) : « étaient présents, de la part de l'Eglise Orthodoxe des Etats-Unis, leurs Eminences le métropolite Nicolas de la Métropole Grecque de Détroit, le Métropolite Christophore de la Métropole Serbe $»{ }^{26}$ Des petites modifications interviennent au niveau de la structure des nominations employées par des acteurs religieux supérieurs à ceux qui sont nommés (ou désignés). Le titre honorifique disparaît, il ne reste plus que le titre passe-partout Monseigneur-, suivi du prénom et de la dignité ecclésiastique du prélat désigné en question: "Dans ce moment historique, nous prions notre Seigneur Jésus-Christ... de bénir l'âme et le cœur du nouvel Pasteur, Mgr. Jean Cassien de Vicina, évêque vicaire de l'Archevêché Orthodoxe Roumain des Etats-Unis et du Canada " - extrait du message de Sa Béatitude le Patriarche Théoctiste à l'occasion de l'ordination épiscopale du père Jean Cassien Tunaru. ${ }^{27} \mathrm{Ce}$ type particulier de discours renferme également un exemple de formule d'auto-nomination, qui est composée du prénom du prélat, suivi de ses fonctions ecclésiastiques hiérarchiques : « avec ma bénédiction paternelle, Théoctiste, archevêque de Bucarest, Métropolite de Munténie et de Dobrogea, locuntenens de la Césarée de Cappadoce et Patriarche de l'Eglise Orthodoxe Roumaine $» .^{28}$ Les formules d'autonominations des autres acteurs religieux de cette famille hiérarchique sont construites de la même façon. Ce qu'on peut constater est le fait que ces nominations ne font plus référence à un système de parenté, mais à un système hiérarchique et hiérarchisé, à l'intérieur duquel le statut privilégié de ces prélats repose sur de la soumission acceptée, comprise en termes d'obéissance. On arrive ainsi au type particulier de relations interpersonnelles définies par les interpellations utilisées à l'égard des prélats de l'Eglise orthodoxe, en langue française.Les aspects qui relèvent du niveau relationnel son nombreux et diversement traités dans les études faites dans la perspective des approches énonciatives et pragmatiques. Dans le cas précis des interpellations concernant les prélats de l'Eglise orthodoxe, deux sont les dimensions mises en évidence : la dimension horizontale de la distance (plutôt lointaine, affective, mais non familière et non intime) et celle verticale du pouvoir hiérarchique. ${ }^{29}$ Les marques discursives du fonctionnement de ces deux types de dimensions interpersonnelles sont la personne et le nombre du 
pronom, ainsi que les titres honorifiques qui font partie de la structure de ces interpellations. Voyons surtout le fonctionnement énonciatif des formes pronominales. Les acteurs très initiés (dans le sens d'une très bonne connaissance de ces codifications qui existent au niveau des nominations et des interpellations, qui peuvent être des clercs, des moines ou des laïques), qui participent à une interaction communicationnelle de type hiérarchique ascendant, actualisent l'accroche discursive et communicationnelle par l'emploi du pronom votre, suivi de la deuxième partie de la nomination, récupérée au niveau de l'interlocution et transformée de la sorte en interpellation: sainteté, béatitude, éminence, et, respectivement, excellence. C'est une forme d'interpellation très proche -du point de vue formel, grammatical (morphologique), de la nomination. Il s'agit d'une forme élaborée, très cérémonielle, qui va de pair avec le faste des cérémonies religieuses orthodoxes pontificales, caractérisées de la sorte justement à cause de leur célébration par ces acteurs religieux privilégiés (du fait de leur position ecclésiastique hiérarchique) les prélats de l'Eglise orthodoxe (le scénario de ces cérémonies représente justement les "données contextuelles", le cadre externe de l'interaction, qui caractérisent le type particulier de relation hiérarchique entre les interactants, dont on parlait plus haut ${ }^{30}$ ). L'emploi exact de ces interpellations prouve -comme on le disait déjà- le niveau d'initiation terminologique des acteurs religieux et laïques orthodoxes et leur consentement à participer à la mise en scène de cette hiérarchie acceptée et assumée de la sorte. Dans ce cas très précis, l'interpellation des prélats construit en même temps le sujet interpellateur comme un je, ainsi que l'autre (l'acteur interpellé), comme un vous (marque formelle de cette dimension de la distance obéissante et respectueuse), en tant qu'interlocuteur, auquel on reconnaît néanmoins un statut particulier, du fait de son appartenance à une hiérarchie ecclésiastique, transformé en interlocuteur au niveau de l'accroche dans le discours par le pronom de la deuxième personne du pluriel, sous la forme possessive votre. L'interpellation relève donc de l'énonciation, de l'interlocution, de la récupération de ces acteurs religieux en tant qu'interlocuteurs. A la suite de Benveniste, l'énonciation est classiquement définie comme la "mise en fonctionnement de la langue par un acte individuel d'utilisation". ${ }^{31}$ En linguistique du discours, le terme d'interlocuteur est employé pour désigner, au pluriel, les partenaires d'un acte d'échange verbal, en situation de communication orale, chacun prenant successivement la parole. ${ }^{32}$ Compte tenu de la nature particulière de la relation interpersonnelle dont on parlait avant, l'interpellation est engendrée par la concertation énonciative du je et du vous (et non pas du tu). Selon E. Benveniste, je désigne "l'individu qui énonce la présente instance de discours contenant l'instance linguistique je". ${ }^{33}$ Défini par rapport à je, le tu est "l'individu allocuté dans la présente instance de discours contenant l'instance linguistique tu". ${ }^{34}$ Pour ce qui est des nominations, le je définit en désignant le il, une troisième personne, doublée des formes pronominales son, ou sa, que nous avons rencontrées plus haut. Les caractéristiques les plus importantes de cette troisième personne sont: de "n'être jamais réflexive de l'instance de discours; de comporter un nombre parfois assez grand de variantes pronominales ou démonstratives; de n'être pas compatible avec le paradigme des termes référentiels tells que ici, maintenant, etc.". ${ }^{35}$ Dans le cas précis de nos interactions de type hiérarchique ascendant avec des prélats, le je définit le vous et respectivement, la forme votre. Dans le cadre énonciatif défini par un ici et un maintenant de ces interactions précises, le je-doublé du moi- accroche de façon énonciative le vous, doublé de la forme votre. Ce cadre énonciatif est actualisé lors des interactions du type des cérémonies liturgiques, des réunions synodales, des conférences religieuses, des cérémonies d'intronisation d'un prélat, d'ordination à la prêtrise, des 
cérémonies de commémoration historique, manifestées dans des espaces publics ou liturgiques. Le je désigne plus fréquemment des acteurs religieux tels des clercs et de moines, et moins souvent des laïques, qui viennent plus rarement en contact avec des prélats. Les formes d'interpellation employées à l'égard des évêques, des archevêques, et des métropolites par des laïques sont identiques -Monseigneur, même si les manières de les nommer sont différentes. Les acteurs religieux, les clercs et les moines, utilisent des interpellations plus complexes, qui contiennent la forme pronominale votre, suivi du titre honorifique excellence, respectivement, éminence, sans contenir le prénom des prélats interpellés.Dans le cas précis du patriarche, le primat de l'Eglise orthodoxe, l'interpellation reprend aussi une partie de la forme de sa nomination: Sa Béatitude, le patriarche Daniel se verra accroché dans une interaction communicationnelle "initiée"par l'interpellation Votre Béatitude. Dans le cas très précis de ces prélats de l'Eglise orthodoxe, l'interpellation ne comprend pas le nom de ceux-ci. En principe, il s'agit de leur prénom, qui ne se retrouve qu'au niveau de la nomination directe (dans des occasions sociales et liturgiques très précises: pendant des cérémonies de commémoration historique ou des réunions synodales, etc.) ou rapportée (ou indirecte: lorsqu'ils sont évoqués en leur absence physique). Dans ces derniers cas, on assiste à la construction du vous comme un autre, du fait de son évocation par le je.

Les clercs :L'interpellation d'un père de paroisse et d'un hiéromoine (un moine prêtre) par des laïques (orthodoxes) se fera de façon légèrement différente. Les formes correctes permettent d'identifier leur utilisateur selon son niveau d'initiation dans la pratique de l'orthodoxie: mon père et, respectivement, révérend père ou très révérend père (s'il s'agit, comme nous l'avons déjà vu, d'un archimandrite ou du supérieur d'un monastère ${ }^{36}$ ), jamais "Monsieur le prêtre" ou "Monsieur le Curé". Dans le cas des clercs aussi, l'emploi de ces interpellations par les fidèles orthodoxes (des laïques, donc) engendre la mise en place d'une reconnaissance de leur statut ecclésiastique privilégié (justifié et défini par leur qualité sacramentelle de ministres) et d'un consentement à vivre à l'intérieur d'une hiérarchie très bien établie au sein de l'Eglise. On retrouve donc la relation de la paternité spirituelle, dans son acception très largement répandue dans le christianisme en général, la représentation étant celle du berger -père qui garde et s'occupe de ses brebis-enfants, qui lui obéissent. ${ }^{37}$ L'appellatif " père » est gardé au niveau de l'interpellation employée par les clercs entre eux, lors de plusieurs types d'interactions : des rencontres sociales en dehors de l'église, des concélébrations (pendant lesquelles, ils s'invitent à accomplir certains rôles liturgiques), des réunions synodales, etc. La structure de l'interpellation est dans ce cas formée de l'appellatif "père» (qui fixe son statut sacerdotal, tout en le reconnaissant, en tant que marque d'acceptation de la part du je interpellateur de ce statut défini à l'intérieur d'un système ecclésiastique fondé sur la reconnaissance des charismes du ministère), suivi du prénom (donc du nom de baptême) du prêtre en question. La même forme d'interpellation est employée également par les supérieurs hiérarchiques du prêtre, lors des mêmes types d'interactions communicatives: "père Marc ». Les deux différences à noter entre les structures de ces formules d'interpellation sont la disparition du possessif «mon », qui instaure en l'assumant par le je énonciateur et initiateur de l'interpellation la relation de paternité (dans le cas des fidèles, des interactions hiérarchiques ascendantes) et l'apparition du nom du clerc interpellé dans le deuxième cas, celui des interactions hiérarchiques descendantes. En ce qui concerne les formes des nominations des clercs, elles sont consignées par plusieurs types de sources: des journaux paroissiaux, des lettres pastorales, des compte-rendus de réunions synodales ou de conférences, etc. Elles ont une première partie identique, indifféremment 
si le prêtre désigné est un curé ou un moine, constitué de l'appellatif "père ", suivi de son prénom. Ensuite, selon le type de diffusion du texte où apparaissent ces nominations, autrement dit du degré de familiarisation du destinataire de celui-ci avec le prêtre nommé, on précise le nom de famille du père et son titre de ministre (qui indique sa fonction sacerdotale) : «recteur de la paroisse sainte Geneviève ", « hiéromoine dans le monastère de Saint-Antoine-Le-Grand », « archimandrite », etc. Enfin, les simples fidèles de l'Eglise orthodoxe sont nommés entre eux par leur prénom (leur vrai nom de chrétiens baptisés), suivi parfois par leur nom de famille et interpellés par d'autres laïques, ainsi que par des clercs et des prélats, de la même façon : par leur prénom. Evidemment, cette formule d'interpellation relève d'un certain type de relation interpersonnelle définie par rapport à ceux-ci, une relation de " proximité ", de familiarité, fraternelle (définie avec les autres fidèles) et, respectivement, filiale (définie avec les clercs et les prélats, qui les connaissent -ce qui est souvent le cas en France, où les communautés orthodoxes sont importantes, mais pas aussi nombreuses qu'en Roumanie).

\subsection{La mise en scène de quelques rôles liturgiques des ministres}

11 Certaines interpellations et nominations participent aussi à la mise en scène de quelques rôles liturgiques, lors de la célébration des offices de l'Eglise. Pour des raisons d'économie et d'importance énonciative, nous nous rapporterons ici exclusivement à la liturgie eucharistique attribuée à saint Jean Chrysostome, la plus connue par l'orthodoxie en général, car la plus fréquemment célébrée. Le rôle liturgique et symbolique du prêtre, de ministre ordonné sacramentellement qui peut -seul- célébrer l'eucharistie est souligné plusieurs fois par l'énoncé du chœur (accompagné par l'assemblée des fidèles) qui comprend une formule d'interpellation déjà mentionnée à son égard, qui l'invite à actualiser le geste de la bénédiction, au début et à la fin de l'office : « Père saint bénit ! ». La mise en scène de ce rôle symbolique et liturgique du prêtre est sous-tendue par la signification du geste de la bénédiction, de transmettre la paix et la grâce divine sur le(s) destinataire(s) du geste, étant accompli par le prêtre en tant que symbole même du Christ (symbolique sous-tendue par la codification des positions des doigts de sa main droite qui accomplit le geste, qui reconstituent les initiales et les finales grecques du nom du Christ : I, C, X , C) ${ }^{38}$ Il n'y a que le prêtre qui peut bénir les fidèles, en tant que «transparent et symbole du Christ $» .^{39}$ En fait, théologiquement et symboliquement, c'est le père céleste qui bénit l'assemblée eucharistique, à l'intérieur de l'interaction très précise et très complexe de la liturgie, par l'intermédiaire du geste du prêtre, le père terrestre de ses fidèles (qui lui reconnaissent ce statut par leur présence même à l'office liturgique). Quant à lui, le prêtre, une forme d'auto-nomination reconnaît -avec humilité (grande vertu chrétienne !) son statut de serviteur du Christ, instrument humain à l'œuvre dans l'orientation et l'acheminement de ses fils spirituels vers le Royaume du Christ, son maître : «le pécheur que je suis". ${ }^{40}$ Cette forme d'auto-nomination est employée à deux moments précis du scénario liturgique: au moment de la grande entrée (quand le prêtre transfère les saints dons de la table du proscomédiaire sur l'autel, en passant par les portes centrales de l'iconostase), ainsi qu'au moment de l'épiclèse, lorsqu'il invoque la descente du Saint-Esprit sur les saints dons afin que ceux-ci deviennent les espèces consacrées, le sang et le corps du Christ : "Nous t'offrons donc ce culte spirituel et non sanglant, nous t'invoquons, nous te prions et nous te supplions: envoie ton saint Esprit sur nous et sur ces dons. Le prêtre et le diacre s'inclinent par trois fois, disant intérieurement: O Dieu, purifie le pécheur que je suis et prends pitié de moi." ${ }^{41}$ Dans le 
premier contexte (celui de la grande entrée), cette formule d'auto-nomination est accompagnée de deux autres: "l'inutile serviteur que je suis" et "tout indigne servant et pécheur que je suis". ${ }^{42}$ Voyons l'ensemble de ce contexte très précis d'emploi de ces autonominations: "Je t'en supplie donc, toi le seul bon et le seul bienveillant, abaisse ton regard sur le pécheur,sur l'inutile serviteur que je suis; purifie mon cœur et mon âme de toute pensée mauvaise; et moi qui suis revêtu de la grâce du sacerdoce, rends-moi capable, par la puissance de ton saint Esprit de me tenir devant ta sainte table afin d'y consacrer ton Corps saint et sans tache et ton sang précieux. Devant toi je me tiens et j'incline la tête pour te supplier : ne détourne pas de moi ton visage..., mais permets que ces dons te soient offerts par moi, tout indigne servant et pécheur que je suis. ${ }^{43}$ Nous avons là un type particulier d'auto-nomination, actualisée lors d'une interaction spéciale (rituelle liturgique), dans un contexte immédiat de prières de supplication adressées à Dieu, par le prêtre, en tant que serviteur sacerdotal de celui-ci, investi par la grâce sacramentelle de l'ordination, circonscrit par deux contextes plus larges et très importants du point de vue symbolique -la préparation des dons pour la consécration et, respectivement, la consécration des espèces eucharistiques. A ces moments précis du scénario liturgique, la conscience de la contiguïté avec le sacré engendre chez le prêtre ces formules très humbles d'auto-nomination, en tant que reconnaissance de sa nature humaine avant tout (et donc, soumise au péché) de serviteur du Christ, qui est appelé à accomplir le sacrifice suprême (central du christianisme), eucharistique, non sanglant. La composante humaine (ou, en termes théologiques, son hypostase de fidèle-participant à la liturgie) est reconnue et assumée de la sorte par le prêtre même, à côté de ses hypostases de ministre et de symbole du Christ ${ }^{44}$ et l'actualisation de son rôle liturgique essentiel -celui d'accomplir le sacrifice eucharistique- en dépend étroitement.

Nous devons mentionner également le fait qu'en dehors de la participation de ces quelques interpellations et nominations à la mise en scène des rôles liturgiques mentionnés ${ }^{45}$, lors de la célébration des offices de l'Eglise orthodoxe, on assiste à d'autres formes de participations sémiotiques: du gestuel, de l'appropriation et des configurations de l'espace liturgique, qui se constituent (avec les codifications vestimentaires) en autant de manifestations communicationnelles des acteurs liturgiques participants.

\section{BIBLIOGRAPHIE}

Balan, Ioannichié, père (2003). Le Père Cléopas, traduit du roumain par le hiéromoine Marc, préface de Mgr. Daniel, métropolite de la Moldavie et de Bucovine, introduction de Jean-Claude Larchet. Lausanne. L'Age d'Homme, collection « Grands spirituels orthodoxes du XXe siècle ».

Benveniste, E. (1966). Problèmes de linguistique générale: I. Paris : Gallimard.

Benveniste, E. (1974). Problèmes de linguistique générale : II. Paris : Gallimard.

Charaudeau, P., Maingueneau, D. (2002). Dictionnaire d'analyse du discours. Paris : Seuil. 
Deseille, PèrePlacide (1965). L'évangile au désert : L'Evangile au désert des premiers moines à Saint Bernard, présentation, choix de textes et traduction par Placide Deseille -moine de Bellefontaine. Paris : Cerf.

Deseille, Placide (2003). La Spiritualité Orthodoxe et la Philocalie. Paris: éditions Albin Michel.

Dumas, Felicia (2000). Gest şi expresie în liturghia ortodoxă: Studiu semiologic. Iaşi: Institutul European.

Dumas, Felicia (2008). Lexicologie française. Iåi :Casa editorială Demiurg.

Jaubert, Anna (1990). La lecture pragmatique. Paris: Hachette.

Le Tourneau, Dominique (2005). Les mots du christianisme : catholicisme, protestantisme, orthodoxie. Paris : Fayard.

Samuel, hiéromoine (2008). Petit guide des monastères orthodoxes de France. Monastère de Cantauque.

Špidlík, T. (1997). Spiritualitatea rãsãritului cre ${ }^{\circ}$ tin.1. Manual sistematic. traducere ${ }^{\circ} i$ prezentare: diacon Ioan I. Icã jr., cuvînt înainte P. Marko, I.Rupnik S.J. Sibiu: Deisis.

Stãniloae Dumitru (1986). Spiritualitate şi comuniune în liturghia ortodoxă. Craiova: Editura Mitropoliei Olteniei.

Sources:

Feuillet Saint Jean Cassien, « Bulletin hebdomadaire d'information de la Métropole orthodoxe Roumaine d'Europe Occidentale et Méridionale. »

La Divine Liturgie de saint Jean Chrysostome (1986). Rome : Diaconie Apostolique.

\section{NOTES}

1. Son excellence Monseigneur Marc Alric, évêque vicaire de la Métropole Orthodoxe Roumaine d'Europe Occidentale et Méridionale; Son Eminence Monseigneur Joseph Pop, archevêque et métropolite de la Métropole Métropole Orthodoxe Roumaine d'Europe Occidentale et Méridionale.

2. Le père archimandrite Placide Deseille, higoumène (supérieur) du monastère Saint-Antoine-LeGrand, Font de Laval, 26190, St. Laurent-en-Royans, France, métochion du monastère athonite de Simonos Petra. Le terme archimandrite désigne un titre honoriphique accordé à un prêtre -moine, et, en même temps, le supérieur d'un monastère orthodoxe.

3. La mère Hypandia, supérieure du monastère de la Protection de la Mère de Dieu, Mas Solan, 30330, La Bastide d'Engras, France.

4. Felicia Dumas, Lexicologie française, Casa editorială Demiurg, Iaşi, 2008.

5. Arrivés en France en très grand nombre après leur exode d'Asie mineure en 1922, les immigrés grecs ont continué à pratiquer leur foi en langue grecque, leur statut juridictionnel devenant de plus en plus officialisé. Ainsi, les paroisses grecques dépendent-elles directement du Patriarcat œcuménique de Constantinople, ou sont constituées -depuis 1963- dans la Métropole grecque de France. La plupart d'entre elles se trouvent dans le Sud-Est de la France. Les Russes émigrés ont constitué un archevêché russe pour l'Europe occidentale, placé sous la juridiction du Patriarcat œcuménique de Constantinople. Un autre groupe d'émigrés russes se trouvent sous la juridiction de leur Patriarcat d'origine, celui de Moscou. Ceux d'entre eux qui ont refusé cela, considérant que le Patriarcat de Moscou s'était montré beaucoup trop flexible et tolérant à l'égard du pouvoir soviétique, se trouvent sous la juridiction d'un synode d'évêques «hors- 
frontières ». Evidemment, il y a en France des orthodoxes roumains, serbes, bulgares, etc. Mais, il y a aussi des orthodoxes français de souche, des Français convertis à l'orthodoxie. Ceux-ci sont organisés en paroisses qui dépendent juridictionnellement de quelques métropoles -réparties selon les régions géographiques de la France- soumises au Patriarcat de Constantinople. En 2000, Michel Evdokimov parlait de 200000 fidèles orthodoxes en France. Il y en a encore plus à l'heure actuelle, approximativement 300 000, selon le chiffre mentionné par le hiéromoine Siméon dans Petit guide des monastères orthodoxes de France, Monastère de Cantauque, 2008.

6. Cf. E. Benveniste, Problèmes de linguistique générale I, Paris, Gallimard, 1966, p. 254.

7. Cf. Adresse (termes d'), article signé par C. Kerbrat-Orecchioni, dans Dictionnaire d'analyse du discours, sous la direction de P. Charaudeau et D. Maingueneau, Paris, Seuil, 2002, p. 31.

8. Idem, p. 30.

9. Idem, ibidem.

10. Idem, p.31.

11. Cf. Anna Jaubert, La lecture pragmatique; D. Perret, "Les appellatifs, analyse lexicale des Actes de parole", Langages 17.

12. Cf. Père Ioannichié Balan, Le Père Cléopas, traduit du roumain par le hiéromoine Marc, préface de Mgr. Daniel, métropolite de la Moldavie et de Bucovine, introduction de Jean-Claude Larchet, Lausanne, l'Age d'Homme, 2003.

13. Par interaction, nous comprendrons ici une rencontre communicationnelle qui engendre et dirige un échange communicatif dans un lieu et lors d'une occasion donnés. Par interaction hiérarchique ascendante, nous comprenons une rencontre communicationnelle qui occasionne un échange communicatif engendré et dirigé par un interactant de condition hiérarchique inférieure qui se rapporte à un interlocuteur de condition hiérarchique supérieure. En principe, en milieu monastique, ce type d'interactions ont lieu quotidiennement, lors des rencontres à l'intérieur du monastère, au travail, au réfectoire, dans l'église, etc.

14. Le cénobitisme est le mode de vie monastique le plus répandu, de type communautaire, sous l'autorité d'un supérieur appelé higoumène. L'idiorrythmie est une "forme de vie religieuse de groupes de moines vivant ensemble, à l'ombre d'un grand monastère, à leur propre rythme, en conservant une propriété individuelle. » (D. Le Tourneau, Les mots du christianisme: catholicisme, protestantisme, orthodoxie, p. 320). Plus d'une vingtaine de monastères de cette confession orientale existent à l'heure actuelle en France, partagés entre plusieurs juridictions: cinq monastères de la Métropole Grecque, qui dépendent du patriarcat œcuménique, un monastère qui appartient au patriarcat d'Antioche, deux monastères et une skite de l'Archevêché Russe (qui dépendent du patriarcat œcuménique), deux autres monastères et une skite de l'Archevêché Russe (qui dépendent du patriarcat de Moscou), cinq monastères et une communauté monastique de la Métropole Roumaine de l'Europe Occidentale et Méridionale (qui dépendent du patriarcat de Bucarest) et, enfin, deux monastères de l'Evêché Serbe, qui dépendent du patriarcat de Serbie (Hiéromoine Samuel, Petite guide des monastères orthodoxes de France, Monastère de Cantauque, France, 2008, p. 82).

15. Cf. T. Špidlík, T., Spiritualitatea rãsãritului cre ${ }^{\circ}$ tin.1. Manual sistematic, traducere ${ }^{\circ}$ i prezentare: diacon Ioan I. Icã jr., cuvînt înainte P:Marko I.Rupnik S.J., Sibiu, Deisis, p. 193.

16. Hiéromoine Samuel, Petite guide des monastères orthodoxes de France.

17. Balan, Père Ioannichié, Le Père Cléopas, préface de Mgr. Daniel, introduction de Jean-Claude Larchet, traduit du roumain par le hiéromoine Marc, Lausanne, L'Age d'Homme, collection Grands spirituels orthodoxes du XXème siècle, 2003.

18. Cf. PèrePlacide Deseille, L'Evangile au désert des premiers moines à Saint Bernard, Paris, Cerf, 1965, p.40.

19. Idem, p. 36.

20. Cf. D. Le Tourneau, Les mots du christianisme : catholicisme, protestantisme, orthodoxie, p. 594. 
21. «Une place importante est tenue, comme il se doit, par son séjour au Mont Athos et sa rencontre avec saint Silouane, son staretz» (il s'agit d'un livre qui raconte la vie et l'oeuvre théologique du père Sophrony, fondateur de la communauté monastique Saint-Jean Baptiste de Maldon, Essex) : Feuillet Saint Jean Cassien, no 265-266, p. 10.

22. Cf. Père Placide Deseille, La spiritualité orthodoxe et la philocalie, Albin Michel, 2003, p. 17.

23. Feuillet Saint Jean Cassien, no 278, septembre 2007, page 6.

24. Feuillet Saint Jean Cassien, no 267, septembre 2006, p. 20.

25. FSJC, no265-266, p. 23,fragment d'un article de journal signé par une paroissienne de New York (Lavinia Ecaterina Barbu).

26. Idem, p.23.

27. FSJC, no 265-266, p. 25.

28. Idem, ibidem.

29. Cf. Dictionnaire d'analyse du discours, p. 499.

30. Idem, ibidem.

31. Cf. E. Benveniste, Problèmes de linguistique générale II, Paris, Gallimard, 1974, p. 80.

32. Cf. Dictionnaire d'analyse du discours, p. 326.

33. Cf. E. Benveniste, Problèmes de linguistique générale I, Paris, Gallimard, 1966, p. 252.

34. Idem, p.253.

35. Idem, p. 256.

36. Cette dernière forme d'interpellation est identique avec la nomination des archimandrites : « Son Eminence l'Archevêque Nathaniel propose un nouvel évêque vicaire du Diocèse orthodoxe roumain d'Amérique : le très révérend archimandrite Joseph (Morris). » (FSJC no 219, p. 8).

37. Les origines de l'obéissance dans l'orthodoxie sont en liaison avec un père spirituel ; c'est après, qu'on a assisté dans l'Eglise à l'élargissement au domaine juridique, l'obéissance par rapport au charisme du père spirituel se déplaçant également vers le supérieur juridique, prêtre de paroisse, supérieur d'un monastère, évêque, archevêque, métropolite, patriarche : cf. T. Spidlik, op. cit., p. 317.

38. Cf. Felicia Dumas, Gest şi expresie în liturghia ortodoxă. Studiu semiologic , Iaşi, 2000, p. 120.

39. Cf. Dumitru Stăniloae, Spiritualitate şi comuniune în liturghia ortodoxă.

40. Cf. La liturgie de saint Jean Chrysostome, p.47.

41. Idem, ibidem.

42. Idem, p. 37.

43. Idem, ibidem.

44. Cf. Felicia Dumas, Gest şi expresie în liturghia ortodoxă. Studiu semiologic , Iaşi, 2000, p. 115.

45. Pour des raisons d'économie, nous nous sommes arrêtée exclusivement au prêtre, en tant que ministre principal de la célébration liturgique, en laissant de côté le diacre et les prélats, dont les rôles liturgiques sont pratiquement les mêmes.

\section{RÉSUMÉS}

Nous nous proposons de montrer de quelle façon les différentes formes d'interpellation et de nomination employées à l'intention et à l'égard des différents acteurs monastiques, des clercs, des prélats et des simples fidèles dans l'orthodoxie mettent en scène les rôles religieux à l'intérieur d'une hiérarchie ecclésiastique et monastique, et, respectivement, les rôles 
liturgiques, lors de la célébration des offices de l'Eglise. Nos références porteront sur les termes employés dans l'orthodoxie française. Nous analyserons ces différentes formes d'interpellation et de nomination au niveau de leurs emplois différents dans: 1. des fragments de discours et plusieurs types d'interactions verbales enregistrées et 2 . des textes écrits de natures différentes (où ils sont plutôt nommés : des lettres monastiques, des ouvrages de catéchèse, et d'autres). Nous étudierons également le fonctionnement de l'interpellation dans le déclenchement de certains rôles liturgiques du prêtre, lors de la célébration de la liturgie eucharistique (la messe de l'Eglise orthodoxe). De la même façon, on essaiera d'analyser le fonctionnement de quelques formes d'auto-nomination du même acteur liturgique -le prêtre-, qui engendrent une mise en scène de son rôle liturgique principal.

In this paper, we intend to show how different forms of address and salutation used towards monks, clerics, prelates and worshippers in the Orthodox world represent religious roles within the ecclesial and monastic hierarchy as well as liturgical roles during religious service celebration. We will refer to words used in the French Orthodoxy. We will analyse these different forms of address and salutation with respect to their use in: 1. fragments of discourse and several types of recorded verbal interaction and 2. different types of written texts (where people are most of the time addressed: such as monastic letters, catechesis work etc.). We will also analyse the way these forms of address function and cause certain liturgical roles of the priest, during Eucharistic liturgy (the service of the Orthodox Church). We will also try to analyse the functioning of certain forms of self-appointment of the same liturgical actor - the priest - that create a mise-en-scene of his main liturgical role.

\section{INDEX}

Keywords : Forms of address and salutation, orthodoxy, laymen and religious actors, religious and liturgical roles

Mots-clés : nomination, orthodoxie, acteurs religieux et laïques, rôles religieux et liturgiques

\section{AUTEUR}

\section{FELICIA DUMAS}

Université « Alexandru Ioan Cuza » de Iaşi 